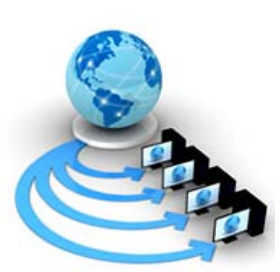

Volume 9, No. 1, January-February 2018

International Journal of Advanced Research in Computer Science

REVIEW ARTICLE

\author{
Available Online at www.ijarcs.info
}

\title{
ACTIVE AND ASSISTED LIVING: A COMPREHENSIVE REVIEW OF ENABLING TECHNOLOGIES AND SCENARIOS
}

\author{
Manoj T \\ Department of Computer Science \\ SMVITM, Bantakal, Udupi \\ Karnataka, India
}

\author{
Dr.Thyagaraju G S \\ Department of Computer Science \\ SDMIT, Ujire, Dakshina Kannada \\ Karnataka, India
}

\begin{abstract}
From the past few decades entire world is witnessing the phenomenon of population ageing as a result of life longevity and declining birth rate of modern society. India is also not immune to this demographic change and will have major socio-economic consequences over the period of time. To extend the necessary assistance for the older adults to live independently and lead a healthy lifestyle the role of Information and Communication Technologies (ICT) is of paramount in nature. One such ICT-based innovative technology is Active and Assisted Living (AAL). In this paper we present comprehensive survey to monitor the recent trends in the realm of AAL. First we discuss about the generic overview of AAL and Ambient Intelligence (AmI). Next, we highlight the relevance of enabling technologies for AAL. Then we review the various trending scenarios of AAL and major research projects being carried out across the world. Finally, we conclude by proposing some possible directions for the future work in the area of AAL.
\end{abstract}

Keywords: Active and Assisted Living, Ambient Intelligence, Human Activity Recognition, Mobility Pattern Analysis, Wearable Sensors, Ambient Sensors, Deep Learning.

\section{INTRODUCTION}

The dwindling birth and mortality rate among the human population has led to the global phenomenon of population ageing. This demographic transition will be more severe and will have a consequential impact on our society in years to come. Most of the developed countries across the globe have already attained the older population age structures than ever seen and heard in the past [1]. In the year 2017, World Population Prospects (WPP) reported that around $13 \%$ of the global population comprising an estimated 962 million in the world are aged 60 or over in the world. The population aged 60 or above is growing at a rate of about $3 \%$ year [2]. According to WHO reports [3], developing countries are witnessing the variation in the pattern of their population attaining the older age structures with number of people aged 65 or older is projected to grow from an estimated 524 million in 2010 to approximately around 1.5 billion in 2050. As per the demographic reports [4], there are nearly 104 million people aged 60 or above in India which comprises the $8.6 \%$ of total population which has increased over time from 5.6\% in 1961 and it is projected that aged population will grow to $20 \%$ (i.e. around 324 million)by 2050 . The surge in the older population will be accompanied with the rapid increase in age-related chronic disorders such as mental health disturbances, cardiovascular diseases; respiration related issues, diabetes, cancer etc. as well as aged-people with physical or cognitive impairments such as sensory loss, motor handicap, hyperactivity disorder etc. According to [5], around 70\% of aged adults with some kind of cognitive or physical impairments cannot stay independently and yearn for the activity assistance from the dependents, healthcare experts or caregivers and about 19 million of population offers primary assistance to their older or dependent relatives to perform their daily activities. In most of the cases, the caregivers to elderly people are family members especially household women or meagerly paid domestic help where they lack the skills to deal with specific cases of age related problems which apparently leads to poor quality of services rendered. In order to alleviate and effectively counter the repercussions of soaring aging population the need of the hour is to improve the living condition of aged people by developing low-cost, secure, reliable, robust, eco-friendly assisted healthcare solutions. Information and Communication Technology (ICT) will play a pivotal role to conquer this upheaval task. During recent decades, the developing applications on ICT-enabled support for independent living of older adults and disabled people has been drawing great attention from the research communities and policy makers. The term of Active and Assisted Living (AAL) has opened up new avenue to innovate cutting edge technologies to provide assistance as well as healthcare and rehabilitation to elder people with physical or mental impairment [6]. To acquire the ambient intelligence from the operational vicinity to monitor the human activity and vital health signs, to travel from one place to another, social inclusiveness, gesture recognition, comprehension of natural language, gait analysis, sensor data analysis from the various data sources suggest the need for confluence of multiple assistive techniques.

This paper is organized into sections as follows: Section II provides an introduction to the Active and Assisted Living (AAL) as well as Ambient Intelligence (AmI). Section III discusses the enabling technologies and infrastructure support for AAL. The Section IV focuses on the critical review of various scenarios in AAL and Section $V$ describes some of the research projects. Section VI concludes the paper by throwing some light on the future directions of AAL. 


\section{AAL AND AMBIENT INTELLIGENCE}

Active and Assisted Living is an emerging multidisciplinary field aiming at exploiting information and communication technologies in personal healthcare and smart health systems to effectively the counter the growing elderly population where users can interact with people in a natural way, wherever and whenever they are needed, being aware of the context (spatial, temporal, emotional etc.) of the user or the environment, and act proactively.

Ambient Intelligence (AmI) is a new paradigm in information technology aimed at empowering people's capabilities by the means of digital environments that are sensitive, adaptive, and responsive to human needs, habits, gestures, and emotions [7].The evolution of these increasingly intelligent smart spaces will facilitate innovative human-machine interactions through ubiquitous, non-intrusive and seamless communication. The true potential of AmI can be realized when it is incorporated with the assistive technologies in the healthcare domain which will completely revolutionize elderly and disabled care.

In order to tackle the challenge of the rendering the quality healthcare services and to provide an independent living environment of their preference to the aged people assistive technologies research and projects were initially conceived as Ambient Assisted Living in the year 2008 by European Framework Program. Later it was renamed as Active and Assisted Living in the year 2014 for the second phase of research funding.

The AAL programme was initiated with the precise set of objectives as follows:

i. To assist the elderly population to stay independently by increasing their autonomy, selfconfidence and mobility.

ii. To offer the healthcare and rehabilitation services to the elderly individuals.

iii. To promote the better and healthier lifestyle for the individuals at risk.

iv. To prevent the aged community from social isolation.

v. To support the caregivers, families and other healthcare personnel to discharge their responsibilities in a flexible manner.

vi. To enhance the efficiency and productivity of used resources in ageing societies.

AAL system is the synergy of multiple technologies such as ubiquitous computing, wireless technologies, sensor technology, Internet of Things (IoT), context awareness, machine learning, cognitive agents and artificial intelligence as shown in Fig.1.

With the upsurge in use of digital devices embedded with sensors, miniaturization of electronic components and seamless connectivity has made ubiquitous computing a reality in AAL environment. The ambient intelligence data is gathered using the electronic, mobile or smart devices are transmitted across the ecosystem of AAL through the advanced wired or wireless networking technologies. The development of intelligent cognitive systems in AAL will be bolstered by machine learning techniques and artificial intelligence. The adaptive and dynamic nature of ambient assistance expects the context awareness to play decisive role in AAL. AAL paradigm can be realized through the
IoT, where the elderly live in their homes with smart objects, thus smart homes, communicating to the outside world in an intelligent and goal-orientated manner.

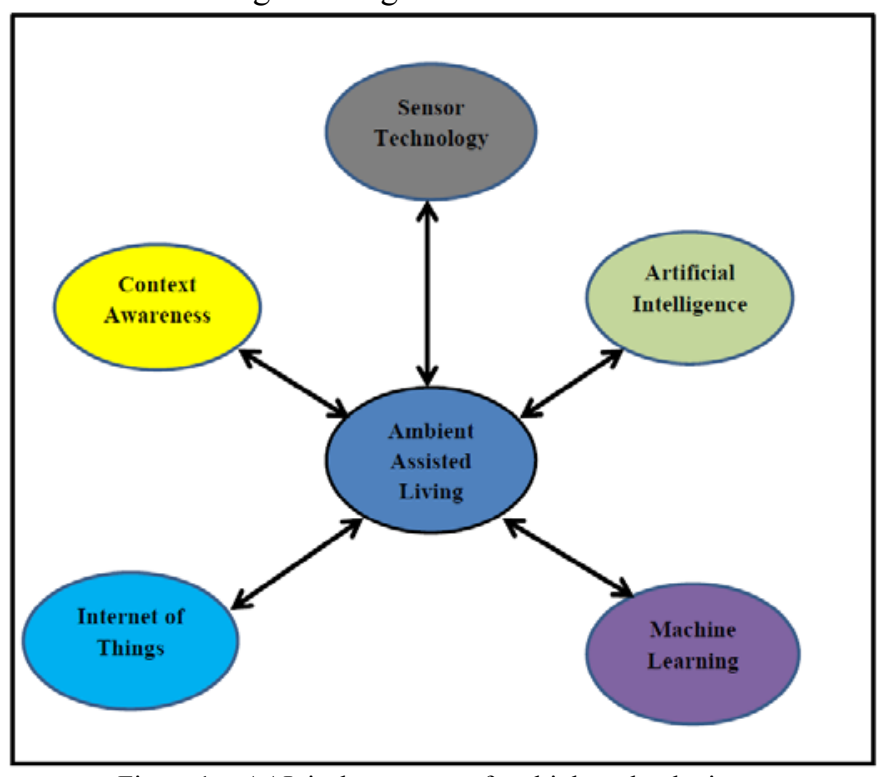

Figure 1. AAL is the synergy of multiple technologies

\section{ENABLING TECHNOLOGIES FOR ACTIVE AND ASSISTED LIVING}

The assisted living is fueled by the incremental advancements in the technologies which aid in development of assistive technologies. Assistive technology is a hypernym referring to specialized and categorized technology used by people to adapt how specific tasks are performed. Assistive technology includes low-tech devices, such as eyeglasses or walkers, as well as high-tech devices, such as hardware, software, and peripherals that assist people with disabilities in accessing computers or other information technologies [8]. In this section we will discuss some key disruptive technologies which will bring the remarkable changes in the development of assistive technologies augmented by ambient intelligence.

\section{A. Sensor Technology}

The widespread use of wireless technologies and the constant miniaturization of the electronic components have given the ample space for the sensors to be embedded in almost all the day-to-day devices and tools. Since assistive technologies have a greater role to play in daily life of ageing population in recent time's sensors presence has become almost indispensable in healthcare domain.

The assistive technologies makes use of most commonly used type of sensors such as temperature, accelerometers, gyroscopes, blood pressure, pulse rate, respiratory rate etc. which are tangible in nature (physical sensors) and can be embedded within the wearables devices like smart watches, phones, fitness bands, clothing and fabrics or even implanted under the skin. But today interpretation of sensors are not limited to a physical sensors but it can also be extended to virtual or logical sensors that do not actually produce data but it may retrieve data from many sources and publish it as sensor data i.e from Facebook or Twitter statuses about environmental data in the city, electronic health records of aged people or service providers information from the databases, email and chat applications 
etc. The effective combination of both physical and virtual sensors in assisted living offers the numerous new, pragmatic and innovative applications for independent and confident living of the aged people by continuously monitoring health features such as heartbeat, glucose level, body temperature, physical activity, blood pressure, ECG (electrocardiogram), EEG (electroencephalography).

\section{B. Context Awareness}

The term context aware computing has its origin in ubiquitous or pervasive computing. It originally refers to location awareness, but more generally means awareness of changes in the environment that are otherwise static, such as the identity, or activity and time. Any information that characterizes the situation of an entity is called context. An entity may be a person, place or object that is considered important to the interaction between a user and an application [9].

As the computing requirement in assistive technologies arise anywhere, anytime due to very nature of its application the context awareness is most expected feature in these applications. In computer science, context awareness, also called sentient, refers to devices that can sense and react to their environment. If the awareness is towards persons, it is called Ambient Intelligence (AmI). AmI refers to computers that are aware of and responsive to the presence of people. It builds on context awareness and is a form of human-centric computer interaction.

From the AAL perspective context awareness is required in accomplishing various tasks such as heart rate monitoring, medication prompting, generation of agenda reminders, temperature changing, weather alerts, emergency notifications, and mood detection etc. In order to achieve this, assistive technologies must have the thorough knowledge of the ambient environments. The context management of the data collected from heterogeneous devices in ambient spaces involves four phases such as context acquisition, context modelling, context reasoning and context dissemination. First of all, context must be acquired from various resources. The sources could be physical sensors, virtual sensors or logical sensors (context acquisition). Second, the collected data needs to be modelled and represent according to a meaningful manner (context modelling). Third, modelled data needs to be processed to derive high-level context information from low-level raw sensor data (context reasoning). Finally, both high-level and low-level context needs to be distributed to the consumers who are interested in context (context dissemination). The techniques used to acquire context can be varied based on responsibility, frequency, context source, sensor type, and acquisition process [10]. Chen and Kotz[11] as well as Strang and Linnhoff-Popien [12] has surveyed no of popular context modelling techniques which are very much relevant to pervasive computing environment such as AAL. Each technique has its own strengths and weaknesses. The six most popular context modelling techniques are: key-value, markup schemes, graphical, object based, logic based, and ontology based modelling. The most popular context reasoning techniques was surveyed by Perttunen et al. [13] as well as Bettini et al. [14]. They classified them into six categories: supervised learning, rules, fuzzy logic, unsupervised learning, probabilistic reasoning and ontological reasoning.

\section{Internet of Things}

Internet of Things (IoT) is most promising technology of the decade which comes with the vision of "Available anywhere, anytime, by anything and anyone". It is the global and dynamic network infrastructure for information society with the self-configuring capabilities based on standards and interoperable communication protocols. IoT has the deep rooted ties with the Ubiquitous computing means the general ability of objects to communicate (anywhere and anytime); Pervasive computing means the enhancement of objects with processing power (the environment around us becomes the computer); AmI means the capability of objects to register changes in the physical environment and thus actively interact in a process [15].

Even though IoT can offer lot of solutions in the AAL domain the two targeted areas where IoT role may be paramount in nature are: communication and data management. In assistive technologies the communication of information may span across different types of networks such Personal Area Network (RFID, NFC, Bluetooth, Zigbee), Local Area Network(WiFi), Metropolitan Area Network(WiMax), Cellular Networks(GPRS,3G,4G,5G) or Wide Area Network(Internet) where the interplay between these communication networks are meticulously handled by IoT. Communication systems in Active and Assisted Living (AAL) platforms have to deal with a huge variety of data types, that can be coarsely classified as behavioral (or habits related),physiological, environmental (or ambient-related), and healthcare data. Such a variety adds further complexity to data management in AAL, that has to cope also with other aspects, such as the amount of data that an AAL platform is able to collect from heterogeneous data sources [16]. IoT will address the issue of interoperability among the heterogeneous assistive tools through the standards developed by vendor community or businesses.

An AAL scenario is characterized by being connected, context-sensitive, personal, adaptive and anticipative. The IoT is supposed to being capable of providing all characteristics necessary for an ambient assisted environment. With respect to the fields of needs for elderly and disabled population it is possible to accomplish all fields through the IoT. The chronic disease management (health, activities of daily living), alarming systems (security),fall detection(safety), reminder services (peace of mind) and enabling people-to-people communication for instance with relatives (social inclusion) without are just a few mentionable applications of AAL through the IoT.

\section{Machine Learning}

AAL gives more importance to the user, the idea of human-centered design and the intelligence needed to allow the system to anticipate needs of the user. This shapes the decision-making of these systems in a different way and can be summarized as: "a digital environment that proactively, but sensibly, supports people in their daily lives" [17]. Most of the assistive technologies currently available for elderly people requires the user to go through short learning curve about how to use the technology but the introduction of machine learning to AAL will be provide a path breaking solutions where environment will learn how to react to the actions and needs of the user, and this will be achieved in an unobtrusive and transparent manner. Thus, the ability to 
learn patterns of behavior becomes an essential aspect for the successful implementation of AAL systems.

A typical scenario in AAL such as human activity recognition, mobility planner for disabled people, drug discovery, facilitating the daily activities, health parameter predictions which were carried out using rudimentary techniques previously are now leveraged by the learning based algorithms. Applying machine learning algorithms for learning based on the collected data is a composite part of AAL systems. The machine learning algorithms can be broadly classified into: supervised learning, unsupervised learning and semi-supervised learning. In order to enhance precision and accuracy of the learning based systems, deep learning based approaches are more appropriate. Deep learning is a class of machine learning techniques that exploit many layers of non-linear information processing for supervised or unsupervised feature extraction and transformation, and for pattern analysis and classification [18]. Deep learning approaches have the ability to process the images/videos/text in their raw forms and automate the process of feature extraction, representation, and classification.

\section{E. Artificial Intelligence}

Since the birth of modern computing, around the fifties, scientists and doctors alike were always captivated by the potential that artificial intelligence (AI) methodologies might have had in medicine and health care applications. The conventional AI methods such as expert systems, knowledge representation techniques have been strongly exploited for designing and implementing medical applications belonging to the following areas: diagnose, prognosis, medical training and so on. The design of intelligent assistive technologies is strengthened by the artificial intelligent methods.

The main aim of AAL systems is to reduce the burden of relatives and care givers of the elderly people by making them to stay independent. The cognitive systems built on AI methods are capable of reasoning, learning and decision making which will alleviate the burden of aged people on the society. The intelligent agents which are human-like will assist the aged people in carrying out the daily activities, understanding the natural language, providing safety and security.

\section{SCENARIOS IN ACTIVE AND ASSISTED LIVING}

The term "Active and Assisted Living" opens up a new avenue to innovate cutting edge solutions for the various scenarios encountered while offering the assistive technologies to the elderly people suffering from physical and mental impairments. The aged people suffering from the chronic disorders requires caregivers, old age homes, hospitals, doctors to recognize their physical activities and monitor their vital health parameters. In addition to that, mobility patterns have to be analyzed to detect the deviations in the health, provide rehabilitation as well as social inclusion. Fig. 2 gives the general scenarios in ALL for elderly care and independent living.

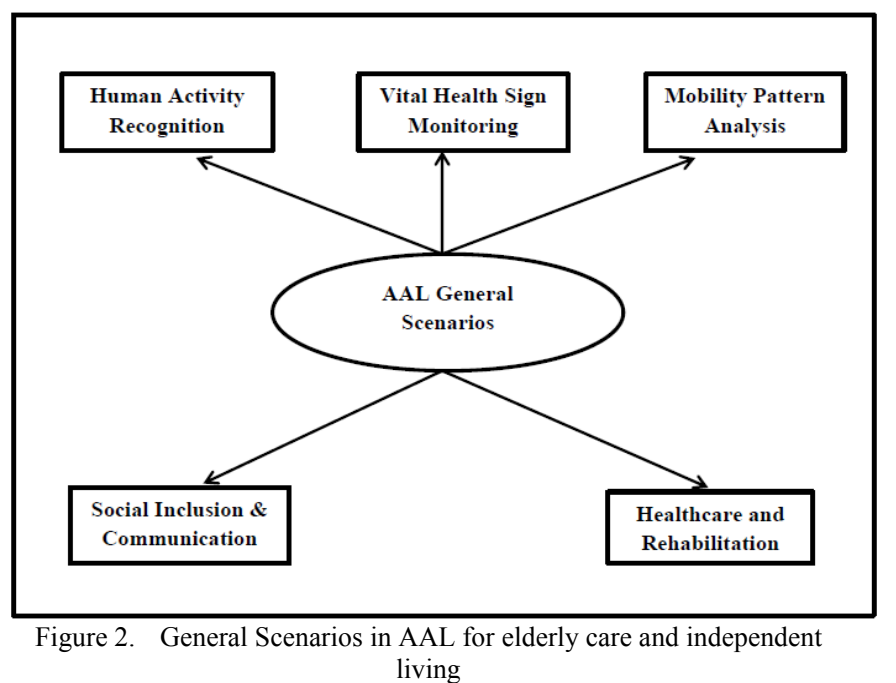

A. Human Activity Recognition

One of the most frequently encountered scenarios in the Active and Assisted Living is the Human Activity Recognition (HAR) of the aged seniors within their dwelling spaces. A HAR component is responsible for recognizing human activity patterns from various types of data collected from AAL devices. The approaches for recognizing the human activity can be classified into vision based approach and sensor based approach as shown in the Fig. 3 .

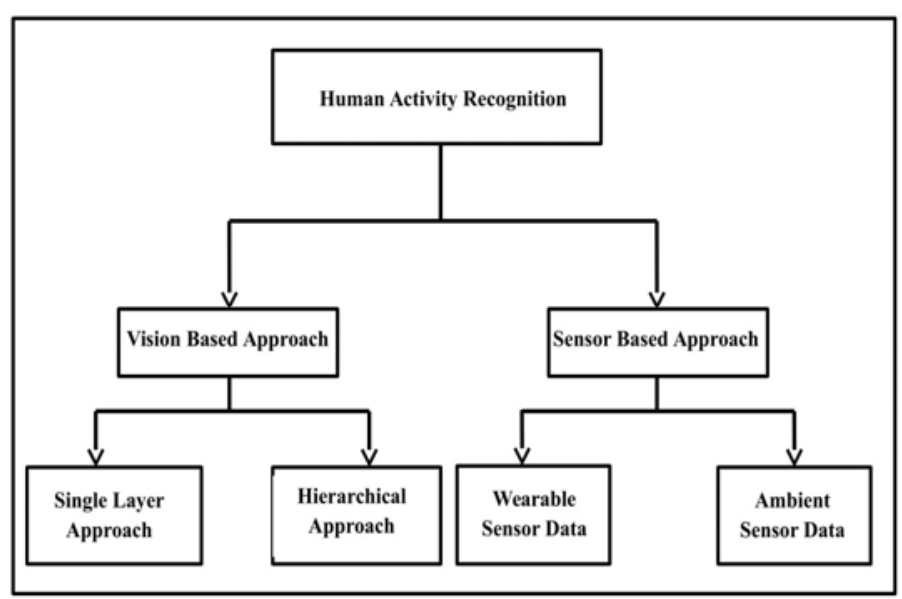

Figure 3. Broad Classification of Human Activity Recognition

The vision based activity recognition which involves the use of visual sensing facilities, such as video recorders and cameras, to observe an object's behavior and environmental changes. Exhaustive context information is obtained from the vision based activity recognition. Typically, the activities are recognized from preprocessed video frames by applying techniques such as foreground-background segmentation [19]. According to [20] vision-based activity recognition techniques consist of two general categories: single layer approaches and hierarchal approaches. Singlelayered approaches recognize human activities directly from a sequence of images, and are more appropriate for recognizing gestures or simple actions. Hierarchical approaches represent high-level human activities in terms of simpler activities or actions; therefore, such techniques are appropriate for recognizing more complex activities. A more detailed survey on vision-based approach can be seen in [20] and [21].

The second category of human activity recognition is sensor based activity recognition which makes use of 
constantly evolving sensor network technologies. Before dwelling into the sensor based activity recognition there is a need to understand the difference between the terms "action" and "activities". The simple behavior executed by an individual which typically lasts for short durations of time is usually referred to as "action". Examples of actions include opening a door, sitting, picking up object from the table, bending, walking etc. On the other hand, complex behaviors consisting of a sequence of actions and/or interleaving or overlapping actions are termed as "activities". They could be performed by an individual or several of them who are required to interact with each other in a certain manner. Examples of activities include many people eating together, two persons preparing a meal etc. [19].

From past two decades the thought of using sensors for activity recognition has been existent amongst research community. The initial breakthrough was provided by the experimental work of the Neural Network house [22] in the context of home automation. Later it was used by researchers extensively in ubiquitous and pervasive computing, smart appliances and activity recognition. A new sensor based approach started to appear in early 2000s, which makes use of sensors embedded into devices for monitoring human activities . The sensitive issues in AAL such as data security and privacy, obtrusiveness, ethics can be handled better in sensor based approach rather than traditional vision based approaches. Our review focuses on two most important sensor based activity recognition approaches such as wearable sensor based and ambient sensor based.

1) Activity Recognition based on wearable sensor data

Wearable sensors are generally a category of sensors that are worn on the body, implanted directly under skin or indirectly carried with the help of individual's body. The signals are generated as the user performs the activities. As a result, person's physiological state or movement can be monitored by these sensors. Wearable sensors can be fixed into clothes, belts, bags, shoes, goggles, headbands, wristwatches, mobile devices, or even implanted on the body. The most frequently used wearable sensor for monitoring activity is accelerometer. They are particularly suitable in situations where repetitive body motions, such as walking, running, standing, sitting and climbing stairs are involved. Many researchers have extensively worked on accelerometer data for activity recognition in various environments. In AAL environment the location based activity recognition will play a pivotal role as aged people with independent living may change their location based on certain circumstances. For monitoring the location based activities seamlessly in open pervasive and ambient environment Global positioning system (GPS) sensors are widely used as wearable sensor. Table I classifies various research papers based on the type of wearable sensor used, the activity recognized by them and machine learning/deep learning methodology or algorithms adopted by those specific works.

Table I. Review of human activity recognition based on wearable sensor data

\begin{tabular}{|c|c|c|c|}
\hline Reference & Types of Sensor used & Activities Recognized & Methodology/Algorithm \\
\hline Yang et al.,2008 [23] & Accelerometer & $\begin{array}{l}\text { Walking, Running, Standing, } \\
\text { Working, Scrubbing }\end{array}$ & Neural classifier algorithm \\
\hline Bonomi et al.,2009 [24] & Accelerometer & $\begin{array}{l}\text { Lying, Sitting, Standing, } \\
\text { Working on a Computer, } \\
\text { Walking, Running, Cycling }\end{array}$ & Decision Tree classifier \\
\hline Ryder et al,2009 [25] & Accelerometer and GPS & Mobility Monitoring & Decision Tree \\
\hline Reddy et al.,2010 [26] & Accelerometer and GPS & $\begin{array}{c}\text { Transport mode between two } \\
\text { places }\end{array}$ & $\begin{array}{l}\text { Decision tree and Discrete } \\
\text { Hidden Markov Model }\end{array}$ \\
\hline Gjoreski et al.,2011 [27] & Accelerometer & $\begin{array}{l}\text { Lying, Sitting, Standing, All } \\
\text { Fours, Transitional }\end{array}$ & $\begin{array}{c}\text { Supervised learning techniques } \\
\text { such as Naive Bayes, SVM, J48 } \\
\text { and Random Forest. }\end{array}$ \\
\hline Chamroukhi et al.,2013 [28] & Accelerometer & $\begin{array}{c}\text { Stairs Ascent and Descent, } \\
\text { Walking, Sitting, Standing Up, } \\
\text { Sitting on the Ground }\end{array}$ & $\begin{array}{c}\text { Multiple regression model } \\
\text { incorporating a hidden discrete } \\
\text { logistic process. }\end{array}$ \\
\hline Bayat et al.,2014[29] & Accelerometer & $\begin{array}{l}\text { Slow Walking, Fast Walking, } \\
\text { Running, Stairs-Up, Stairs- } \\
\text { Down, and Dancing }\end{array}$ & $\begin{array}{l}\text { Individual classifiers such as } \\
\text { Multilayer Perceptron, } \\
\text { Random Forest, LMT, SVM, } \\
\text { Simple Logistic and Logit Boost } \\
\text { are used. }\end{array}$ \\
\hline Moncada-Torres et al.,2014 [30] & Accelerometer & $\begin{array}{c}\text { Sixteen various daily living } \\
\text { activities }\end{array}$ & kNN Classifier \\
\hline Gupta et al.,2014 [31] & Accelerometer & $\begin{array}{l}\text { Walking, jumping, running, } \\
\text { sit-to-stand/stand-to-sit, } \\
\text { stand-to-kneel-to-stand, } \\
\text { and being stationary }\end{array}$ & Naïve Bayes and kNN classifier \\
\hline Zeng et al.,2014 [32] & Accelerometer & $\begin{array}{c}\text { Six daily living activities, } \\
\text { Activities of assembly line } \\
\text { workers in a car maintenance } \\
\text { scenario }\end{array}$ & Convolutional Neural Networks \\
\hline Alsheikh et al.,2015 [33] & Accelerometer & $\begin{array}{c}\text { Six daily living activities, } \\
\text { Activities of assembly line } \\
\text { workers in a car maintenance } \\
\text { scenario }\end{array}$ & $\begin{array}{l}\text { Deep Learning and Hidden } \\
\text { Markov Models }\end{array}$ \\
\hline Jiang et al.,2015 [34] & Accelerometer and Gyroscope & Walking, Sitting, Lying, Standing & $\begin{array}{c}\text { Deep Convolutional Neural } \\
\text { Networks }\end{array}$ \\
\hline Wang et al.,2017 [35] & $\begin{array}{l}\text { Wireless Body-Worn Sensor } \\
\text { Nodes }\end{array}$ & $\begin{array}{c}\text { Location, Activity and Gesture } \\
\text { Recognition }\end{array}$ & Convolutional Neural Networks \\
\hline
\end{tabular}




\section{2) Activity recognition based on ambient sensors}

Even though wearable sensor based approaches can obtain satisfactory performance, it is difficult for them to be widely applied in AAL environment because this kind of method requires users to wear or carry corresponding sensors all the time which may intrusive in nature while performing the daily activities and in some scenarios system may encounter with the task of recognizing the complex activities which may involve group of individuals engaged together to accomplish some task. For ex : Multiple people involved in cooking activity, aged person's interaction with pet animals etc. To recognize more complex activities, a network of ambient sensors is used to model resident activities in the environment, as a sequence of sensor events. Here the term "ambient sensors" stands for goal specific sensors embedded in various devices or tools used in daily living environment which will interact with humans or other objects of similar type. Table II provides the review of some research papers on activity recognition through multiple ambient sensors.

Table II. Review of Human activity recognition based on ambient sensor data

\begin{tabular}{|c|c|c|c|}
\hline Reference & Types of Sensor used & Activities Recognized & Methodology/Algorithm \\
\hline Wang et al.,2009 [36] & Ambient Sensors & $\begin{array}{c}\text { User movement, user location, } \\
\text { human-object interaction, } \\
\text { human-human interaction and } \\
\text { environmental information }\end{array}$ & $\begin{array}{c}\text { Coupled Hidden Markov } \\
\text { Model(CHMM) }\end{array}$ \\
\hline Cook et al.,2012 [37] & Ambient Sensors & Multiple activities & $\begin{array}{c}\text { Semi-supervised and supervised } \\
\text { learning techniques }\end{array}$ \\
\hline Suryadevara et al.,2014[38] & Ambient sensors & Activities of Daily Living & $\begin{array}{c}\text { Sensor activity pattern } \\
\text { algorithm }\end{array}$ \\
\hline Krishnan et al.,2014 [39] & Ambient Sensors & $\begin{array}{c}\text { Eleven daily living activities } \\
\text { Machines[SVM] }\end{array}$ & $\begin{array}{c}\text { Support Vector } \\
\text { Atomic activity, complex } \\
\text { activity and multi-level complex } \\
\text { activity }\end{array}$ \\
\hline Liu et al.,2016 [40] & Ambient Sensors & & Shapelet based approach \\
\hline
\end{tabular}

\section{B. Vital Health Sign Monitoring}

The independent living of the elderly people suffering from chronic disorders or mental impairments demands the need for unobtrusive assistive technologies in monitoring their vital health signs such as Blood Pressure (BP), Body Temperature (BT), Respiratory Rate (RR), Pulse (Heart Rate), Blood Glucose level, Gait Speed etc. for immediate medical intervention, in case of emergency which may otherwise result into fatal consequences. The Vital health sign monitoring allows the aged seniors to stay at home and obtain the medication rather than in expensive healthcare facilities such as hospitals or nursing homes. It thus provides an alternative in the form of home healthcare to the aged community instead of on-site clinical monitoring. Such systems provisioned with unobtrusive and non-invasive wearable sensors can be feasible diagnostic solution to the healthcare personnel for monitoring significant physiological signs and activities of the patients in real-time, from a remote facility [41]. Therefore it is apparent that wearable sensors play a crucial role in vital health sign monitoring that has grabbed the attention of many researchers, academicians, entrepreneurs, and tech giants in recent years. A wide range of application specific health sign monitoring techniques are quoted in literature. Table III provides some of recent algorithms and methodology adopted for health sign monitoring.

Table III. Review of Vital health sign monitoring using sensor data

\begin{tabular}{|c|c|c|c|}
\hline Reference & Types of Sensor used & Vital Health Signs Monitored & Methodology/Algorithm \\
\hline Zheng et al.,2014[42] & Body-Worn Sensors & $\begin{array}{c}\text { Classification of Emotions using } \\
\text { EEG Signals }\end{array}$ & Deep Belief Networks \\
\hline Rahhal et al.,2016[43] & Body-Worn Sensors & Classification of ECG Signals & Deep Neural Networks \\
\hline Khan et al.,2017[44] & Ambient Sensors & Respiration & $\begin{array}{c}\text { Convolutional Neural } \\
\text { Networks }\end{array}$ \\
\hline Acharya et al.,2017[45] & Body-Worn Sensors & Heart Disease from ECG \\
Signals & $\begin{array}{c}\text { Deep Convolutional Neural } \\
\text { Networks }\end{array}$ \\
\hline W.Gu, 2017[46] & Wearable Sensors & Blood Glucose Level & $\begin{array}{c}\text { Deep Recurrent Neural } \\
\text { Networks }\end{array}$ \\
\hline
\end{tabular}

\section{Mobility Pattern Analysis}

Mobility is one of the key components to quality of life as it allows a people to move independently one location to another according to their wish whenever they intend to do so. The AAL environment will encounter the scenario where people with motor disabilities and cognitive impairments are unable to perform the movement to desired destination on their own. As a consequence of this, a considerable chunk of the population suffering from motor impairments must rely upon others for assistance to move from place to place. The mobility pattern analysis of elderly or people suffering from mental disorders at indoor and outdoor locations will provide the multi-faceted advantages for the assistive technologies to build customized healthcare solutions to the individuals. The analysis will have two major advantages

i. Any abnormality in the day-to-day mobility pattern of an elderly person both at home and outdoors may be a manifestation or early symptom of evolving health complications.

ii. The mobility patterns developed over a period of time will help to build the assistive devices which will be able to guide an individual to take right direction while moving from one location to another and help

him/her from getting lost from their normal path.

The assistive systems developed for mobility pattern analysis typically involve the sensor based ecosystem equipped with 
wearable sensors and non-wearable sensors to track the mobility pattern and provide responses when deviations are detected. Mobile devices carried by human generate the mobile data naturally, which it involves the mobility patterns of an individual carrier. Smartphone is most commonly used mobile device embedded with the GPS sensor which collects location context of the user. Song et al. [47] were of the opinion that human mobility is always non-random in nature, which is gives indication that people always appear in several specific places. In spite of its diversity, human mobility always follows the simple reproduction mode and a high degree of predictability. The crux of the human spatial mobility is that it has some special features such as i. Regional - The probability of leaving a small area decreases with time.

ii. Strongly regular and predictable.

From the mobility perspective term location includes both indoor and outdoor locations. The path which is daily traversed by the user from one particular source to destination has to be collected for certain period and analysis has to be carried out using the machine learning or deep learning techniques. Table IV gives the review of research work carried out in the area of human mobility pattern analysis.

Table IV. Review of Human mobility pattern analysis using sensor data

\begin{tabular}{|c|c|c|c|}
\hline Reference & Types of Sensor used & Location Context & Methodology/Algorithm \\
\hline Leightley et al.,2016[48] & $\begin{array}{c}\text { Microsoft Kinect One Depth } \\
\text { Sensor }\end{array}$ & Indoor & $\begin{array}{c}\text { Machine Learning Classifiers } \\
\text { such as SVM, ANN, Random } \\
\text { Forests, GRBM, Adaptive } \\
\text { Boosting, LPBoost, RUSBoost, } \\
\text { Total Boost, Bagging were used }\end{array}$ \\
\hline Tang et al.,2016[49] & $\begin{array}{c}\text { Simulation as well as Camera } \\
\text { Data }\end{array}$ & Indoor & Bayesian Framework \\
\hline Ghourchian et al.,2017[50] & $\begin{array}{c}\text { Ambient Sensors } \\
\text { (WiFi Signals in a room) }\end{array}$ & Indoor & $\begin{array}{c}\text { Semi-Supervised Learning } \\
\text { Framework }\end{array}$ \\
\hline Ouyang et al.,2017[51] & $\begin{array}{c}\text { Location Sensors in Mobile } \\
\text { devices }\end{array}$ & Outdoor & $\begin{array}{c}\text { Deep Convolutional Neural } \\
\text { Networks }\end{array}$ \\
\hline Barlacchi et al.,2017[52] & $\begin{array}{c}\text { Smartphone data(calls, SMSs, } \\
\text { GPS for location) }\end{array}$ & Indoor and Outdoor & $\begin{array}{c}\text { Machine Learning classifiers } \\
\text { such as Logistic Regression, } \\
\text { Random Forest and Gradient } \\
\text { Boosted Trees }\end{array}$ \\
\hline
\end{tabular}

\section{Social Inclusion And Communication}

As all humans are social being they can't remain in isolated environment for the longer period. Social interaction and communication has the significant impact in enhancing the quality of individual's life. As an individual, interacting with others and taking part in social activities and entertainment are the part and parcel of leading a decent life. Traditionally, people can get to communicate with their families and friends through email, phone and various internet based messaging as well as chat platforms(social media) such as Facebook, Twitter, WhatsApp etc. However, many aged seniors with advanced cognitive/physical impairments have hindrance to access the social media and participate in social activities. So another important mission of AAL is to prevent the social isolation of aged as well as disabled people. Table $\mathrm{V}$ gives the description of the AAL applications and research works in the providing Social inclusion and communication to aged people.

Table V. Review of AAL Applications and Research works for Social Inclusion and Communication Table VI

\begin{tabular}{|c|c|}
\hline Reference & Description \\
\hline Gárate et al.,2005[53] & $\begin{array}{l}\text { GENIO project is one of the early AAL application in assisted living for the elderly people. It assists the aged } \\
\text { community to socialize as well as to perform the routine tasks such as reading and analyzing emails, checking for } \\
\text { essentials in refrigerator, operating the washing machine, doing a online shopping in a supermarket with the help } \\
\text { of a PDAs, offering recipes for variety of dishes, programming the dishwasher, watching selected videos and } \\
\text { photos, listening to music etc. }\end{array}$ \\
\hline $\begin{array}{l}\text { Leonardi et } \\
\text { al.,2008[54] }\end{array}$ & $\begin{array}{l}\text { The objective of NETCARITY was to deliver the services that promotes the social connect between the aged } \\
\text { people living in seclusion and the rest of the society with assistive technology as an enabler. The project proposed } \\
\text { an e-inclusion prototype where a user interface enabled with touch-screen technology involves the direct } \\
\text { manipulation of the digital objects in accordance with natural gestures. }\end{array}$ \\
\hline Doyle et al.,2010[55] & $\begin{array}{l}\text { Building Bridge project targeted to enrich the experience of social connectivity for the elderly people with their } \\
\text { peers and families by exploiting the communication technologies. This application opens an avenue for older } \\
\text { people to have a shared experience of a video or radio broadcast with their peers and families. The listeners have } \\
\text { an opportunity to take part in a group chat after the completion of each broadcast. }\end{array}$ \\
\hline $\begin{array}{l}\text { Gómez-Sebastià et } \\
\text { al.,2011[56] }\end{array}$ & $\begin{array}{l}\text { COAALAS [79] builds on the results of two European projects: EU-Share-It (FP6- 045088) and EU-ALIVE } \\
\text { (FP7-215890).It is an intelligent social and norm-aware device for elderly people which is able to autonomously } \\
\text { organize, reorganize and interact with the different actors involved in elderly-care, either human actors or other } \\
\text { devices. It aims to produce a new generation of AmI devices for elderly people by synergizing different } \\
\text { techniques of Artificial Intelligence(AI) such as autonomy, proactivity, social behavior and adaptability. }\end{array}$ \\
\hline $\begin{array}{c}\text { HOST } \\
\text { Project,2014[57] }\end{array}$ & $\begin{array}{l}\text { The HOST project was a part of the EU AAL Joint Programme and a smart technological solution to integrate the } \\
\text { self-service society with the aged seniors in social housing. It aims to offer a healthy and comfortable lifestyle to } \\
\text { older population by bringing the more value adds to the services delivered to them by self-service society. }\end{array}$ \\
\hline
\end{tabular}

\section{E. Healthcare And Rehabilitation}

Health plays a very critical and decisive role in the life of elderly people who have been agonized from chronic diseases such as dementia, diabetes, asthma and other cognitive or physical impairments. The healthcare and rehabilitation solutions must be simple, safe, secure, non-intrusive and costeffective so that it allays the fear of older people about performing their everyday activities. The elderly generation 
leading a secluded life will have a fear factor in their conscience that, they will be unable to call others for help if they meet with some of accidents at their place of residence. These accidents may include incidents such as thermal burns caused in kitchen, fall in the restroom or other areas within house, safety regarding mobility while moving out of the house. It is therefore the assistive tools which come into the rescue of elderly people in these situations, in case if they cannot call others for their help on their own. With the shrinking support from families, society demands for a better alternative for information processing and tools to rehabilitate the recuperating elders or ensure the well-being of the growing number of elderly citizens. Home healthcare is the one of burgeoning area of investigation in the wake of advances in health and communications technology come at a time of dramatic worldwide increase in life expectancy and skyrocketing health care costs. Home monitoring and assistive technologies are deployed to observe changes in the pattern of health and behavior of older population within their home settings and to facilitate successful adaptation to those changes. Hence many countries and businesses have taken the stock of this situation and funded the research communities for the development of pervasive healthcare systems. Table VI gives provides a brief description of assistive applications available for healthcare and rehabilitation.

Table VII. Review of Assistive applications for Healthcare and Rehabilitation

\begin{tabular}{|c|c|}
\hline Reference & Description \\
\hline $\begin{array}{l}\text { Corchado et } \\
\text { al.,2008[58] }\end{array}$ & $\begin{array}{l}\text { GerAmi (Geriatric Ambient Intelligence)[83] an intelligent assistive platform which facilitates the management } \\
\text { and control of geriatric residences by integrating multi-agent systems, mobile devices, RFID and Wi-Fi } \\
\text { technologies. The assessment of the system was carried out to reduce task time with Doctor agent and Nurse } \\
\text { agent, deliver the patient care by boosting the communication between patients and hospital staff. }\end{array}$ \\
\hline $\begin{array}{l}\text { Cameirão et } \\
\text { al.,2009[59] }\end{array}$ & $\begin{array}{l}\text { The Rehabilitation Gaming System(RGS) a virtual reality(VR) based system which makes use of non-intrusive, } \\
\text { multi-mode stimulation techniques targeted at speeding up the functional recovery of effected parts of the human } \\
\text { body after lesions to the nervous system. The RGS is designed in such a way that the patients are involved in } \\
\text { context specific training scenarios that modify according to their performance allowing for an customized } \\
\text { individual training with varying levels of complexity. }\end{array}$ \\
\hline $\begin{array}{c}\text { Kameas and } \\
\text { Calemis,2010[60] }\end{array}$ & $\begin{array}{l}\text { They have surveyed a bunch of pervasive systems which is involved in providing the healthcare and } \\
\text { rehabilitation services for the well-being of elderly population. @ } \text { HOME is a remote health monitoring system in } \\
\text { which patients are equipped with the ambulatory sensors which will acquire the vital parameters of the body such } \\
\text { as blood pressure, ECG, oxygen saturation level and subsequently pass this information to the PC station at } \\
\text { patient's home via communication networks. }\end{array}$ \\
\hline $\begin{array}{l}\text { Project iCarer, } \\
2012[61]\end{array}$ & $\begin{array}{l}\text { The iCarer project a holistic cloud based care support service that provides the informal care to the older adults } \\
\text { through the inter-operable solutions. The value added services include an individualized support and training } \\
\text { program based on online learning modules, assistance packages for caregivers and monitoring as well as } \\
\text { assistance services for the patient being cared for. }\end{array}$ \\
\hline Khan et al.,2012[62] & $\begin{array}{l}\text { The Home Healthcare Monitoring System (HHMS) collects relevant medical as well as daily routine data of older } \\
\text { adults to enrich their daily life and promote the healthy lifestyle. The data gathered from the HHMS platform are } \\
\text { diagnosed by physicians to check for any early signs of health issues among the older population being } \\
\text { monitored. }\end{array}$ \\
\hline
\end{tabular}

\section{RESEARCH PROJECTS IN ACTIVE ASSISTED LIVING}

Currently, umpteen research projects/programs are being carried out in the domain of AAL by various university research labs, mainstream technological industries and state funded research agencies across the globe. Some of the prominent AAL research groups are:

The Centre for Advanced Studies in Adaptive Systems (CASAS) [63] Smart Home project is a multi-disciplinary research project at Washington State University. It is the convergence of technologies in machine learning and pervasive computing that assist in development of smart environments to create valuable functions such as remote health monitoring, active living and personal healthcare of the aged community. The CASAS platform treats ambient environment as intelligent agents and keeps track of the status of the residents and their physical vicinity with the help of adaptive sensors and depending upon the existing context it will act upon the environment using controllers in a way that leverages safety, comfort and living experience of the residents. The system is lightweight and simple so that its performance can be scaled as per the requirements.

The Ambient Intelligence Research Lab(AIR) [64] at Stanford university concentrates on development of methodologies and applications for assisted living in smart homes and offices using ambient data. In addition to that, targeted groups work on different scenarios of assisted living such as occupancy-aware smart buildings, behavior analysis for aging-in-place, personal recommender systems in smart offices etc. The lab is set up in the form of a smart room giving look and feel of natural living environment while presenting an ambient interface to its users through pervasive sensing, seamless processing and ubiquitous communication.

The European Network of Living Labs (ENoLL) [65] is a consortium of AAL research groups across Europe, with almost 400 historically recognized Living Labs and more than 170 active members today. These research groups publish on

all topics of AAL smart homes, as well as all other aspects of human environments with technology integration.

The existence of ENoLL and the scale of its success demonstrate the pervasive scale and scope of AAL research. The Aware Home Research Initiative(AHRI) [66] at Georgia Institute of Technology is the remarkable project in assisted living providing the impetus to other research work. It addresses a range of health and environmental issues. It includes a dedicated home, a suite of managed eldercare homes, and a laboratory focused on children's development. The systems include sensors: motion, light, temperature, humidity, door and window, and cameras and controls: lights, lamp and appliance modules, and door locks. The notable contribution of the Aware Home group is in 
understanding and building AAL systems for individuals with autism.

The TigerPlace [67] is a healthcare facility developed at University of Missouri with a long history of advancements in AAL technologies. Their melding of nursing and technology established their work as a pioneer in AAL healthcare. The facility is an instrumented eldercare space with a focus on helping people age in place. TigerPlace's research group focuses on sensor issues for deploying systems in healthcare scenarios, detecting illness through behavior, detecting and predicting falls, privacy and perceptions of smart monitoring technologies among older adults.

The Future care lab at RWTH Aachen University[68] is an experimental assisted living project which aims at comprehending user's life at home and analyzing how they interact and communicate with the non-intrusive technology. Within Future Care Lab, the design and development of user-centric smart healthcare solutions are conceived and executed by a dedicated multidisciplinary team of individuals having expertise in wide range of fields such as psychology, communication science, computer science, medicine, engineering, statistics and architecture. It focuses on developing novel approach for designing the integrative models for user-centric solutions in smart home domain. This vision is fueled by the new concepts of electronic monitoring systems within assisted living environment which are custom made to suit individuals.

The Quality of Life Technology (QoLT) Centre[69] is funded by the US National Science Foundation (NSF) Engineering Research Center (ERC) in Pittsburgh. The Center is joint venture between Carnegie Mellon University (CMU) and the University of Pittsburgh. Its prominent focus area is development of intelligent solutions for older population with disabilities to make them live independently as well as happily. The Center addresses the issues by prototyping the personalized assistive robots, profiling cognitive and behavioral patterns, driver assistance and human awareness technologies.

The POSEiDON project's [70] objective is to address the issues of the people suffering from Down's syndrome and provide them the personalized smart healthcare solutions. Research topics include augmented reality tools for daily life, ethical considerations in engineering AAL systems.

ORCATECH Living Labs at Oregon Health and Science University [71] is a longstanding and strong contributor to health focused AAL smart homes. With over 480 homes and eight years of longitudinal data, their results have begun to contribute to many ecological validity studies on health metrics and therapies based on AAL technologies. Their research work focuses on infrastructure topics of sensors and systems, UX issues, clinical metrics, mobility in AAL spaces and cognitive function.

The Intelligent assistive technology and systems lab (IATSL) at University of Toronto [72]. IATSL seeks to develop zero-effort technologies with adaptive AAL systems, notably for occupational therapy and gerontology. The group has been very successful with large-scale, interinstitutional research projects that have enabled significant contributions in many healthcare areas using AAL technologies. The group focuses on Alzheimer's disease, ageing in place, ambient healthcare, rehabilitation, and many other technology-based AAL topics.

\section{CONCLUSION}

In the last decade the sensors and computers have gotten faster, cheaper and smaller which embarked many of the technological evolutions in the field of AAL. But any endto-end AAL solution has the role of multiple technologies in it. The voluminous data is generated and consumed by heterogeneous devices at designated levels of AAL system. In prevailing scenario there is no free flow or exchange of data among the heterogeneous entities which has constrained our efforts in expanding the horizon of AAL solutions and has led to the creation of data silos. One of the major futuristic challenges in this area is to achieve interoperability among the devices of various commercial manufacturers.

The global adoption of the AAL solutions by the users is going at the slower pace than expected even though there many healthcare startups and major tech industries ready to offer the solutions. The use of ambient sensors seems to be more restricted to public spaces rather than its intended use in residential places. But the use of wearable sensors embedded in mobile devices or worn by the user has drawn much needed attention. The major research challenge in near future would be about reducing the power consumption as well as miniaturization of sensors. The most prominent amongst ambient sensors used in assisted living are the video-based devices. They generate lot of information or data from the area of their application such as video surveillance used for public safety, recognition of activities of daily living, recording the mobility patterns both in indoors and outdoors etc. But in the current scenario video based analytics cannot be treated as the robust solution as they as lack reliable use under unrestricted conditions and also doesn't have concrete policies to meet privacy issues.

The wide range of sensors deployed in AAL ecosystem is expected to accomplish their designated roles by acquiring relevant information from the environment and the users at varying levels of reliability. In order to acquire the quality and context rich data all these sensors must work in tandem to reduce the uncertainty of data and develop more accurate AAL solutions. The research in the area of personal assistive robots with cognitive skills will take a giant leap in a days to come where they are able to assist elderly people in performing their everyday activities, monitor and analyze their daily routine and make them to interact with the remote caretakers. As the knowledge required for cognitive robots to interact with the users keeps on changing constantly deep learning models can be exploited in future research. To reduce the burden of computation at edge devices or gateways edge computing technology can be explored.

Another application area of AAL that would require major focus is Human Activity Recognition (HAR). The long term analysis and continuous learning in these systems would be more appropriate as learning user routines and detecting deviations from those routines may pose a huge challenge in multi-occupancy environment where context awareness and fusion of captured data will play a crucial role. 


\section{ACKNOWLEDGEMENT}

We would like to thank the management of SMVITM and SDMIT for providing the excellent infrastructure and required library resources in accomplishing this survey work fruitful

\section{REFERENCES}

[1] B. Mirkin and M. B. Weinberger, "The Demography of Population Ageing," Dep. Econ. Soc. Aff. United Nations Secr., no. January, pp. 8-10, 2010.

[2] U. N. Department of Social and Economic Affairs, "World Population Prospects The 2017 Revision," in World Population Prospects, 2017, pp. 11-12.

[3] R. Suzman and J. Beard, "WHO,Global Health and Aging,Technical Report," Natl. Insititute Aging,National Institutes Heal., vol. 1, no. 4, pp. 273-277, 2011.

[4] National Sample Survey Office, "Elderly In India," in Ministry of Statisitcs and Programme Implementation, Govt of India, 2016, pp. 1-95.

[5] L. a Jacobsen, M. Kent, M. Lee, and M. Mather, “America's aging population," Popul. Bull., vol. 66, no. 1, pp. 1-18, 2011.

[6] R. Li, B. Lu, and K. D. McDonald-Maier, "Cognitive assisted living ambient system: a survey," Digit. Commun. Networks, vol. 1, no. 4, pp. 229-252, 2015.

[7] A. Acampora, Giovanni Cook, Diane Rashidi, Parisa Vasilakos, "A survey on Ambient Intelligence in Health Care," Natl. Inst. Heal., vol. 101, no. 12, pp. 2470-2494, 2014.

[8] B. R. Bryant, D Bryant, AssistiveTechnology For People With Disabilties. Allyn and Macon, Boston,MA, 2003.

[9] A. K. Dey and G. D. Abowd, "Towards a Better Understanding of Context and Context-Awareness," Comput. Syst., vol. 40, no. 3, pp. 304-307, 1999.

[10] C. Perera, A. Zaslavsky, P. Christen, and D. Georgakopoulos, "Context Aware Computing for The Internet of Things," IEEE Commun. Surv. Tutorials, vol. 16, no. 1, pp. 414-454, 2014.

[11] G. Chen and D. Kotz, "A Survey of Context-Aware Mobile Computing Research," Dartmouth Comput. Sci. Tech. Rep., vol. 3755, pp. 1-16, 2000.

[12] T. Strang and C. Linnhoff-Popien, "A Context Modeling Survey,” Work. Adv. Context Model. Reason. Manag. UbiComp 2004 - Sixth Int. Conf. Ubiquitous Comput., vol. Workshop o, no. 4, pp. 1-8, 2004.

[13] M. Perttunen, J. Riekki, and O. Lassila, "Context Representation and Reasoning in Pervasive Computing," Int. J. Multimed. Ubiquitous Eng., vol. 4, no. 4, pp. 1-28, 2009.

[14] C. Bettini et al., "A survey of context modelling and reasoning techniques," Pervasive Mob. Comput., vol. 6, no. 2, pp. 161-180, 2010.

[15] A. Dohr, R. Modre-Osprian, M. Drobics, D. Hayn, and G. Schreier, "The internet of things for ambient assisted living," Inf. Technol. new Gener. seventh Int. Conf., pp. 804-809, 2010.

[16] S. Spinsante, E. Gambi, L. Montanini, and L. Raffaeli, "Data management in ambient assisted living platforms approaching IoT: A case study," in 2015 IEEE Globecom Workshops, GC Wkshps 2015 - Proceedings, 2015.

[17] J. C. Augusto, "Ambient intelligence: The confluence of ubiquitous/pervasive computing and artificial intelligence," in Intelligent Computing Everywhere, 2007, pp. 213-234.

[18] L. Deng, "Deep Learning: Methods and Applications," Found. Trends ${ }^{\circledR}$ Signal Process., vol. 7, no. 3-4, pp. 197$387,2014$.
[19]

L. Chen, J. Hoey, C. D. Nugent, D. J. Cook, and Z. Yu, "Sensor-based activity recognition," IEEE Transactions on Systems, Man and Cybernetics Part C: Applications and Reviews, vol. 42, no. 6. pp. 790-808, 2012.

[20] J. Aggarwal and M. Ryoo, "Human activity analysis: A review," ACM Comput. Surv., vol. 43, no. 3, p. 16:116:43, 2011.

[21] A. Sargano, P. Angelov, and Z. Habib, "A Comprehensive Review on Handcrafted and Learning-Based Action Representation Approaches for Human Activity Recognition," Appl. Sci., vol. 7, no. 1, p. 110, 2017.

[22] M. C. Mozer, "The neural network house: An environment that adapts to its inhabitants," Am. Assoc. Artif. Intell. Spring Symp. Intell. Environ., no. December, pp. 110-114, 1998.

[23] J. Yang, J. Wang, and Y. Chen, "Using acceleration measurements for activity recognition : An effective learning algorithm for constructing neural classifiers," Pattern Recognit. Lett., vol. 29, no. 16, pp. 2213-2220, 2008.

[24] A. G. Bonomi, A. H. C. Goris, B. Yin, and K. R. Westerterp, "Detection of Type, Duration, and Intensity of Physical Activity Using an Accelerometer," Med. Sci. Sport. Exerc, 2009.

[25] J. Ryder, B. Longstaff, S. Reddy, and D. Estrin, "Ambulation: A Tool for Monitoring Mobility Patterns over Time Using Mobile Phones," Comput. Sci. Eng. 2009. CSE '09. Int. Conf., vol. 4, pp. 927-931, 2009.

[26] S. Reddy, M. Mun, J. Burke, D. Estrin, M. Hansen, and M. Srivastava, "Using mobile phones to determine transportation modes," ACM Trans. Sens. Networks, vol. 6, no. 2, pp. 1-27, 2010.

[27] H. Gjoreski, M. Luštrek, and M. Gams, "Accelerometer placement for posture recognition and fall detection," in Proceedings - 2011 7th International Conference on Intelligent Environments, IE 2011, 2011, pp. 47-54.

[28] F. Chamroukhi, S. Mohammed, D. Trabelsi, L. Oukhellou, and Y. Amirat, "Joint segmentation of multivariate time series with hidden process regression for human activity recognition," Neurocomputing, vol. 120, no. July 2014, pp. 633-644, 2013.

[29] A. Bayat, M. Pomplun, and D. A. Tran, "A study on human activity recognition using accelerometer data from smartphones," in Procedia Computer Science, 2014, vol. 34, no. C, pp. $450-457$.

[30] A. Moncada-Torres, K. Leuenberger, R. Gonzenbach, A. Luft, and R. Gassert, "Activity classification based on inertial and barometric pressure sensors at different anatomical locations.," Physiol. Meas., vol. 35, pp. 1245 63, 2014.

[31] P. Gupta and T. Dallas, "Feature Selection and Activity Recognition System Using a Single Triaxial Accelerometer," vol. 0, no. 0, 2014.

[32] M. Zeng et al., "Convolutional Neural Networks for Human Activity Recognition using Mobile Sensors," Proc. 6th Int. Conf. Mob. Comput. Appl. Serv., vol. 6, pp. 197-205, 2014.

[33] M. A. Alsheikh, A. Selim, D. Niyato, L. Doyle, S. Lin, and H.-P. Tan, "Deep Activity Recognition Models with Triaxial Accelerometers," TheWorkshops Thirtieth AAAI Conf. Artif. Intell., pp. 1-8, 2015.

[34] W. Jiang and Z. Yin, "Human Activity Recognition Using Wearable Sensors by Deep Convolutional Neural Networks," in Proceedings of the 23rd ACM international conference on Multimedia - MM '15, 2015, pp. 13071310.

[35] J. Wang, X. Zhang, Q. Gao, H. Yue, and H. Wang, "Device-Free Wireless Localization and Activity Recognition: A Deep Learning Approach,” IEEE Trans. Veh. Technol., vol. 66, no. 7, pp. 6258-6267, 2017. 
[36] L. Wang, T. Gu, X. Tao, and J. Lu, "Sensor-based human activity recognition in a multi-user scenario," in Lecture Notes in Computer Science (including subseries Lecture Notes in Artificial Intelligence and Lecture Notes in Bioinformatics), 2009.

[37] D. J. Cook, "Learning Setting- Generalized Activity Models for Smart Spaces," IEEE Intell. Syst., vol. 27, no. 1, pp. 32-38, 2012.

[38] N. K. Suryadevara and S. C. Mukhopadhyay, "Determining wellness through an ambient assisted living environment," IEEE Intell. Syst., vol. 29, no. 3, pp. 3037, 2014.

[39] N. C. Krishnan and D. J. Cook, "Activity recognition on streaming sensor data," Pervasive Mob. Comput., vol. 10, no. PART B, pp. 138-154, 2014.

[40] L. Liu, Y. Peng, S. Wang, M. Liu, and Z. Huang, "Complex activity recognition using time series pattern dictionary learned from ubiquitous sensors," Inf. Sci. (Ny)., vol. 340-341, pp. 41-57, 2016.

[41] M. J. Deen, "Information and communications technologies for elderly ubiquitous healthcare in a smart home," Pers. Ubiquitous Comput., vol. 19, no. 3, pp. 573$599,2015$.

[42] W.-L. Zheng, J.-Y. Zhu, Y. Peng, and B.-L. Lu, "EEGBased Emotion Classification Using Deep Belief Networks," Multimed. Expo, pp. 1-6, 2014.

[43] M. M. A. Rahhal, Y. Bazi, H. Alhichri, N. Alajlan, F. Melgani, and R. R. Yager, "Deep learning approach for active classification of electrocardiogram signals," Inf. Sci. (Ny)., vol. 345, pp. 340-354, 2016.

[44] U. M. Khan, Z. Kabir, S. A. Hassan, and S. H. Ahmed, "A Deep Learning Framework using Passive WiFi Sensing for Respiration Monitoring," 2017.

[45] U. R. Acharya, H. Fujita, O. S. Lih, M. Adam, J. H. Tan, and C. K. Chua, "Automated detection of coronary artery disease using different durations of ECG segments with convolutional neural network," Knowledge-Based Syst., vol. 132 , pp. 62-71, 2017.

[46] W. Gu, "Non-intrusive Blood Glucose Monitor by Multitask Deep Learning," in 16th ACM/IEEE International Conference on Information Processing in Sensor Networks, Pittsburgh, PA, USA, 2017, pp. 249-250.

[47] C. Song, T. Koren, P. Wang, and A. L. Barabási, "Modelling the scaling properties of human mobility," Nat. Phys., vol. 6, no. 10, pp. 818-823, 2010.

[48] D. Leightley, M. H. Yap, and J. McPhee, "Automated Analysis and Quantification of Human Mobility using a Depth Sensor.," IEEE J. Biomed. Heal. informatics, vol. X, no. X, pp. 1-10, 2016.

[49] B. Tang, C. Jiang, H. He, and Y. Guo, "Probabilistic Human Mobility Model in Indoor Environment," in International Joint Conference on Neural Networks, 2016, pp. 1601-1608.

[50] N. Ghourchian, M. Allegue-Martinez, and D. Precup, "Real-Time Indoor Localization in Smart Homes Using Semi-Supervised Learning," in 29th AAAI Conference on Innovative Applications, 2017, pp. 1-8.

[51] X. Ouyang, C. Zhang, P. Zhou, and H. Jiang, "DeepSpace: An Online Deep Learning Framework for Mobile Big Data to Understand Human Mobility Patterns," pp. 1-11, 2016.

[52] G. Barlacchi, C. Perentis, A. Mehrotra, M. Musolesi, and B. Lepri, "Are you getting sick? Predicting influenza-like symptoms using human mobility behaviors," EPJ Data Sci. A SpringerOpen J., vol. 6, no. 1, 2017.

[53] A. Gárate, N. Herrasti, and A. López, "GENIO: an ambient intelligence application in home automation and entertainment environment," sOc-EUSAI '05 Proc. 2005 Jt. Conf. Smart objects Ambient Intell. Innov. Context. Serv. usages Technol., no. october, p. 241, 2005.
[54] C. Leonardi, C. Mennecozzi, E. Not, F. Pianesi, and M. Zancanaro, "Supporting Older Adults Social Network : the Design of e-Inclusion Communication Services," 2008.

[55] J. Doyle, Z. Skrba, R. McDonnell, and B. Arent, "Designing a touch screen communication device to support social interaction amongst older adults," BCS '10 Proc. 24th BCS Interact. Spec. Gr. Conf., pp. 177-185, 2010.

[56] I. Gómez-Sebastià, D. García-Gasulla, and S. AlvarezNapagao, "Special theme : Ambient Assisted Living," ERCIM NEWS no. 87, pp. 23-24, 2011.

[57] “ABOUT HOST - PROJECT DESCRIPTION.” [Online]. Available: http://www.host-aal.eu/cms/welcomehost/about-host-project-description. [Accessed: 17-Jan2018].

[58] J. M. Corchado, J. Bajo, and A. Abraham, "GerAmi: Improving healthcare delivery in geriatric residences," IEEE Intell. Syst., vol. 23, no. 2, pp. 19-25, 2008.

[59] M. S. Cameirão, S. Bermúdez i Badia, E. Duarte Oller, and P. F. M. J. Verschure, "The Rehabilitation Gaming System: A review," Stud. Health Technol. Inform., vol. 145, no. August 2017, pp. 65-83, 2009.

[60] A. Kameas and I. Calemis, Pervasive Systems in Healthcare:HandBook of Ambient Intelligence and Smart Environments. IOS Press, 2010.

[61] "iCarer | ACTIVE AND ASSISTED LIVING PROGRAMME." [Online]. Available: http://www.aaleurope.eu/projects/icarer/. [Accessed: 17-Jan-2018].

[62] W. A. Khan, M. Hussain, M. Afzal, M. B. Amin, and S. Lee, "Healthcare standards based sensory data exchange for Home Healthcare Monitoring System.," Conf. Proc. IEEE Eng. Med. Biol. Soc., vol. 2012, pp. 1274-7, 2012.

[63] "WSU CASAS | Center for Advanced Studies in Adaptive Systems." [Online]. Available: http://ailab.wsu.edu/casas/. [Accessed: 17-Jan-2018].

[64] "Ambient Intelligence Research Lab." [Online]. Available: http://airlab.stanford.edu/index.html. [Accessed: 17-Jan-2018].

[65] "Open Living Labs | The First step towards a new Innovation System." [Online]. Available: http://www.openlivinglabs.eu/. [Accessed: 17-Jan-2018].

[66] J. A. Kientz, S. N. Patel, B. Jones, E. Price, E. D. Mynatt, and G. D. Abowd, "The Georgia Tech aware home," Proceeding twenty-sixth Annu. CHI Conf. Ext. Abstr. Hum. factors Comput. Syst. - CHI '08, p. 3675, 2008.

[67] M. Fergenson, "TigerPlace: An Innovative 'Aging in Place' Community.,” Am. J. Nurs., vol. 113, no. 1, p. 68, 2013.

[68] "Future Care Lab | Open Living Labs." [Online]. Available: http://www.openlivinglabs.eu/livinglab/futurecare-lab. [Accessed: 17-Jan-2018].

[69] "QoLT Center - QoLT Center - Carnegie Mellon University." [Online]. Available: https://www.cmu.edu/qolt/. [Accessed: 17-Jan-2018].

[70] "About the Project | POSEIDON." [Online]. Available: http://www.poseidon-project.org/about/. [Accessed: 17Jan-2018].

[71] "What We Do | ORCATECH | OHSU." [Online]. Available: http://www.ohsu.edu/xd/research/centersinstitutes/orcatech/about/welcome.cfm. [Accessed: 17Jan-2018].

[72] "IATSL - Intelligent Assistive Technology and Systems Lab.” [Online]. Available: http://iatsl.org/. [Accessed: 17Jan-2018]. 